\title{
Human rhinovirus $C$ infection is associated with asthma in children determined by XTAG respiratory viral panel FAST
}

\section{Dear Editor,}

Cumulative evidence supports the role of early-life viral infections, especially respiratory syncytial virus (RSV) and human rhinovirus (HRV), as major antecedents of childhood asthma (Lemanske, 2002; Jackson et al., 2008). In this study, the XTAG respiratory viral panel FAST (RVP FAST) assay, a multiplex polymerase chain reaction (PCR)-based method (Arens et al., 2010; BaladaLlasat et al., 2011; Gharabaghi et al., 2011; Selvaraju, 2012), was used to investigate the association of infection with different HRV species, especially HRV-C, and the development of asthma in young children living in Beijing, China.

During October 2013 to November 2014, nasopharyngeal aspirates (NPA) for respiratory virus screening from two groups of patients were obtained. The asthma group (147 individuals) who were younger than 16 years of age, and diagnosed with asthma was characterized by recurrent episodes $(\geq 3)$ of wheezing, breathlessness, chest tightness, and coughing, particularly at night or in the early morning, and the control group (81 individuals) was characterized underlying surgical therapy without confirmed respiratory infections within 10 days prior to NPA collection. The mean age of the asthma group was $2.7( \pm 1.5)$ years and $110(74.8 \%)$ were male. The mean age of the control group was $3( \pm 2.3)$ years and $67(82.7 \%)$ were male. Total nucleic acid (DNA and RNA) was extracted using the QIAamp MinElute Virus Spin Kit (Qiagen GmbH, Germany) according to the manufacturer's instructions. Extracts were then tested using the RVP FAST assay simultaneously detecting 16 types of viruses, including influenza A virus (FluA) (subtyped as
$\mathrm{H} 1, \mathrm{H} 3$, or H5), FluB, respiratory syncytial virus (RSV) $A$ and $B$, parainfluenza virus 1-4 (PIV1-4), human adenovirus (HAdV), human metapneumovirus (HMPV), coronaviruses 229E, NL63, OC43, HKU1, enterovirus $(\mathrm{EV}) / \mathrm{HRV}$, and human bocavirus (HBoV) according to the manufacturer's instructions in 96-well plate format (Luminex Molecular Diagnostics Inc., Toronto, Canada).

Among specimens analyzed, 147 from the asthma group and 81 from the control group, 93.0\% (212/228) tested were positive using the RVP FAST assay. The overall proportion of viral infection in the asthma group $(98.6 \%)$ was higher than in the control group $(82.7 \%)$ $\left(\chi^{2}=20.29, P=0.0000007\right)$ (Table 1$)$. In both groups, patients predominantly had a single viral infection $(50.3 \%$ in the asthma group versus $54.3 \%$ in the control group); however, no significant difference was observed between the two groups $\left(\chi^{2}=0.331, P=0.565\right)$. Co-infections were more frequently observed in the asthma group (48.4\%) than in the control group $(28.4 \%)\left(\chi^{2}=8.539, P=0.003\right)$. Similar distributions between the two groups were observed for the majority of detected viruses (Table 2). Enterovirus/HRV was the most common pathogen $(76.9 \%$ and $69.1 \%)$, followed by RSVs $(21.7 \%$ and $9.9 \%)$, PIVs $(13.9 \%$ and $13.6 \%)$, HAdV (15.6\% and $6.2 \%)$, and $\mathrm{HBoV}(13.6 \%$ and $4.9 \%)$ in the asthma and control groups, respectively. No significant difference was observed in the distribution of viral pathogens between the two groups, except for RSVB $\left(\chi^{2}=6.828, P=0.009\right)$ and HMPV $\left(\chi^{2}=6.368, P=0.012\right)$. All HBoVs positive specimens were positive for other viruses (co-infection) (100\%) in both groups, while all HAdV positive specimens were co-infected with other viruses in the asthma group $(100 \%)$. No FluA or B, HMPV, or OC43-positive spec-

Table 1. Viral screening results in different groups using the RVP FAST assay

\begin{tabular}{|c|c|c|c|c|c|c|c|}
\hline \multirow{2}{*}{ Groups } & \multirow{2}{*}{$\begin{array}{l}\text { No. } \\
\text { tested }\end{array}$} & \multirow{2}{*}{$\begin{array}{l}\text { Virus infection positive } \\
\text { No. }(\%)^{a}\end{array}$} & \multirow{2}{*}{$\begin{array}{l}\text { Single virus infection } \\
\text { No. }(\%)^{b}\end{array}$} & \multicolumn{4}{|c|}{ Co-infection No. $(\%)^{c}$} \\
\hline & & & & Two viruses & Three viruses & Four viruses & Five viruses \\
\hline Asthma & 147 & $145(98.6)$ & $74(50.3)$ & $57(38.8)$ & $11(7.5)$ & $2(1.4)$ & $1(0.7)$ \\
\hline Control & 81 & 67 (82.7) & 44 (54.3) & 19 (23.5) & $2(2.5)$ & $1(1.2)$ & $1(1.2)$ \\
\hline
\end{tabular}

Note: $\mathrm{a}: x^{2}=17.9264, P<0.05 ; \mathrm{b}: x^{2}=0.1912, P>0.05 ; \mathrm{c}: x^{2}=7.7371, P<0.05$. 
Table 2. Distribution of viral pathogens in the patient groups determined using the RVP FAST assay

\begin{tabular}{|c|c|c|c|c|c|c|}
\hline \multirow[b]{2}{*}{ Viral type } & \multicolumn{3}{|c|}{ Asthma group $(n=147)$} & \multicolumn{3}{|c|}{ Control group $(n=81)$} \\
\hline & $\begin{array}{l}\text { Total No. } \\
(\%)\end{array}$ & $\begin{array}{l}\text { Single No. } \\
\left(\%^{a}\right)\end{array}$ & $\begin{array}{l}\text { Co-infected No. } \\
\left(\%{ }^{b}\right)\end{array}$ & $\begin{array}{l}\text { Total No. } \\
(\%)\end{array}$ & $\begin{array}{l}\text { Single No. } \\
\left(\%^{a}\right)\end{array}$ & $\begin{array}{l}\text { Co-infected No. } \\
\left(\%^{b}\right)\end{array}$ \\
\hline EV/HRV & $113(76.9)$ & $55(48.7)$ & $58(51.3)$ & $56(69.1)$ & $33(58.9)$ & $23(41.1)$ \\
\hline RSVA & $13(8.8)$ & $6(46.2)$ & $7(53.8)$ & $6(7.4)$ & $3(50.0)$ & $3(50)$ \\
\hline $\mathrm{RSVB}^{\mathrm{c}}$ & 19 (12.9) & $3(15.8)$ & $16(84.2)$ & $2(2.5)$ & 0 & $2(100)$ \\
\hline HBoV & $20(13.6)$ & 0 & $20(100)$ & $4(4.9)$ & 0 & $4(100)$ \\
\hline FluA & $3(2.0)$ & 0 & $3(100)$ & 0 & 0 & 0 \\
\hline FluB & $1(0.7)$ & $1(100)$ & 0 & $1(1.2)$ & 0 & $1(100)$ \\
\hline PIV1 & $1(0.7)$ & 0 & $1(100)$ & $2(2.5)$ & $2(100)$ & 0 \\
\hline PIV2 & 0 & 0 & 0 & 0 & 0 & 0 \\
\hline PIV3 & $12(8.2)$ & $2(16.7)$ & $10(83.3)$ & $4(4.9)$ & 0 & $4(100)$ \\
\hline PIV4 & $7(4.8)$ & $3(42.9)$ & $4(57.1)$ & $5(6.2)$ & $1(20.0)$ & $4(80.0)$ \\
\hline HMPV $^{d}$ & $11(7.5)$ & $3(27.3)$ & $8(72.7)$ & 0 & 0 & 0 \\
\hline HAdV & $23(15.6)$ & 0 & $23(100)$ & $5(6.2)$ & $2(40.0)$ & $3(60.0)$ \\
\hline NL63 & $3(2.0)$ & 0 & $3(100)$ & $4(4.9)$ & $1(25.0)$ & $3(75.0)$ \\
\hline HKU1 & $1(0.7)$ & 0 & $1(100)$ & $3(3.7)$ & 1 (33.3) & 266.7 \\
\hline OC43 & $3(2.0)$ & $1(33.3)$ & $2(66.7)$ & 0 & 0 & 0 \\
\hline $229 \mathrm{E}$ & $2(1.4)$ & 0 & $2(100)$ & $1(1.2)$ & $1(100)$ & 0 \\
\hline
\end{tabular}

Note: a: Single No./Total No.; b: Co-infected No./Total No.; c: $x^{2}=5.63466, P<0.05 ; \mathrm{d}: x^{2}=4.84309, P<0.05$.

imens were detected in the control group, and no PIV2positive specimens were detected in this study (Table 2).

For EV/HRV-positive specimens, a semi-nested RTPCR (targeting the conserved VP4/VP2 region) was performed for HRV confirmation, and the amplified products of the second semi-nested PCR run were sequenced by Invitrogen Inc. for phylogenetic analysis to identify the HRV species (Zhao, 2016). Another RT-PCR was subsequently performed for EV screening of HRV-negative specimens (Zhu, 2008).

Of the $113 \mathrm{EV} / \mathrm{HRV}$-positive specimens in the asthma group, twenty-three specimens were negative in subsequent PCR analyses for EV or HRV screening. Four specimens were positive in EV screening and were later confirmed by sequencing and phylogenetic analysis. Eighty-six specimens were positive in HRV screening: 29 for HRV-A, one for HRV-B, and 47 for HRV-C by sequencing and phylogenetic analysis, and nine were inconclusive because of insufficient PCR products (Table $3)$. However, in the control group, among $56 \mathrm{EV} / \mathrm{HRV}$ positive specimens, eleven specimens were negative in subsequent EV or HRV screening. Nine specimens were $\mathrm{EV}$ positive, including eight confirmed as EV and one as HRV-B by sequencing and phylogenetic analysis. Thirtysix specimens were HRV-positive, including fifteen as
HRV-A, three as HRV-B, ten as HRV-C, and one as EVD68 by sequencing and phylogenetic analysis. The results of seven specimens were inconclusive because of insufficient PCR products (Table 3).

The overall proportion of HRVs in the asthma group $(52.4 \%)$ was higher than that in the control group (35.8\%) $\left(\chi^{2}=5.770, P=0.016\right)$, but no difference was observed in the proportion of HRV-A $\left(\chi^{2}=0.049, P=0.825\right)$ or HRV-B infection $\left(\chi^{2}=2.652, P=0.103\right)$ between the two groups. However, HRV-C infection was more frequently observed in the asthma group than in the control group $(32.0 \%$ vs. $12.3 \%)\left(\chi^{2}=10.729, P=0.001\right)$.

The results shown in the study for viral pathogen

Table 3. HRV species determined using RVP FAST combined with PCR and sequencing in all patients

\begin{tabular}{|c|c|c|c|c|c|}
\hline \multirow{2}{*}{ Groups } & \multirow{2}{*}{ No. tested } & \multicolumn{4}{|c|}{ No. positive (\%) } \\
\hline & & HRV-A & HRV-B ${ }^{c}$ & $\mathrm{HRV}-\mathrm{C}^{\mathrm{d}}$ & Total $^{a}$ \\
\hline Asthma & 147 & $29(19.7)$ & $1(0.7)$ & $47(32.0)$ & $77(52.4)$ \\
\hline Control & 81 & 15 (18.5) & $4(3.7)$ & $10(12.3)$ & 29 \\
\hline
\end{tabular}

Note: $\mathrm{a}: X^{2}=5.12263,0.01<P<0.05 ; \mathrm{b}: X^{2}=0.19119, P>$ 0.05; c: $X^{2}=2.65242, P>0.05 ; \mathrm{d}: X^{2}=9.70824, P<0.05$. 
screening revealed that $82.7 \%$ of the control group had a viral infection, which suggests a high rate of asymptomatic viral infection in children, or some unknown viral infection in children with underlying conditions. Comparison of the viral pathogen results between the asthma group and the control group showed that the overall proportion of viral infections in the asthma group was higher than that in the control group $(P=0.0000007)$, however, no significant difference was observed in the distribution of viral pathogens between the two groups, except for RSVB $(P=0.009)$ and HMPV $(P=0.012)$. Although single viral infection was predominant in both groups, co-infections were more commonly detected using the RVP FAST assay, with a higher frequency observed in the asthma group than in the control group $(P=$ $0.003)$. The highest co-infection frequency was observed with $\mathrm{HBoV}$. These data indicate that the RVP FAST assay can provide more information regarding the pathogenic role of viral infection(s) by distinguishing a single infection from co-infection (Zhu et al., 2014).

Enterovirus/HRV were the most common pathogens detected among the 18 viral or subtype pathogens analyzed by RVP FAST, with a prevalence of $76.9 \%$ (113/ $147)$ and $69.1 \%(56 / 81)$ in the asthma and control groups, respectively. No significant difference was observed in the rate of single or co-EV/HRV infection in either patient group. Although Asner et al. observed increased disease severity among children with single EV/HRV infection compared with children with co-EV/HRV infection (Asner et al., 2015), our study did not support this finding. Further investigation is required to confirm whether such an association exists.

In this study, we also evaluated the pathogenic role of HRV in asthma using the results of HRV subtyping obtained from the RVP FAST assay. Among EV/HRV-positive specimens, most were identified as HRV positive, especially in the asthma group (HRV: $\mathrm{EV}=86: 4$ in the asthma group and $36: 9$ in the control group), indicating that HRV infection was more common than EV infection in the asthma group. However, about $20 \%$ of all patient specimens were inconclusive for EV or HRV positivity following PCR screening because of insufficient PCR products. Several specimens suspected as EV by PCR screening were confirmed as HRV by sequencing, or vice versa, whereas other specimens suspected as HRV by PCR screening showed no sequencing results because of insufficient PCR products. These findings may be explained by the high genetic and antigenic diversity of EV and HRV, with approximately 169 identified HRV types (McIntyre et al., 2013). Thus, development of more sensitive methods is necessary to detect as many EV and HRV types as possible for more precise results.

In this study, a significant difference in the overall proportion of HRVs was observed between the asthma group and the control group $(P=0.016)$, which supports the hypothesis that HRVs are a major trigger for exacerbation of asthma (Miller and Mackay, 2013). As with many other viruses, the specific HRV strain or genotype has an impact on asthma pathogenesis (Moore et al., 2013; Fawkner-Corbett et al., 2016). Using the RVP FAST assay combined with PCR and sequencing, we further subtyped HRV-positive specimens to evaluate their role in asthma. While no significant difference was observed in the rate of HRV-A $(P=0.825)$ or HRV-B infection $(P=0.103)$ between the two groups, HRV-C infection was predominantly observed in the asthma group $(P=0.001)$. Our data suggest that the role of HRV infection in triggering asthma is linked to HRV-C, while HRV-A and -B have little or no impact on development of asthma and are associated with asymptomatic infection.

The present results are in contrast to a previous study that found no significant difference in the prevalence of HRVs, including HRV-C, between asthma and control groups (Zhao et al., 2016). The same semi-nested PCR method followed by sequencing was used for HRV detection in the present study as used in the previous study. In the previous study, TRIzol reagent (Invitrogen Inc., Carlsbad, CA, USA) was used for RNA and DNA extraction, while the QIAamp Min Elute Virus Spin Kit was used in the present study. The discrepancy between two studies may be explained by the differing nucleotide extraction method used between the studies. Xu et al. has demonstrated the dual use of the MS2 and IAC assays in their study highlights the variability of inhibitors present in different lots of green onions and the necessity for removing these compounds prior to detection of the target by real-time RT-PCR. Though the formats for the silica membrane-based QIAamp Min Elute Virus Spin Kits differed slightly in intended input type and volume, they were found to consistently reduce PCR inhibitors to levels which would allow detection of low numbers of MS2 from highly concentrated green onion washes (Xu et al., 2017). Thus more data should be accumulated to confirm the effect of RNA extraction methods on RTPCR.

The RVP FAST assay demonstrates the rate of viruspositive specimens increased to $93.0 \%$ in the asthma group, which supports the theory that viral infection, especially EV/HRV, is one dominant trigger viral pathogen for asthma. While HRV-A and -B likely play an important role in asymptomatic infection, the role of HRV infection in triggering asthma is linked more possibly to HRV-C.

\section{FOOTNOTES}

This study was supported by Special Funds for Public Welfare 
Projects (2015SQ00160), the Beijing Municipal Health Bureau “Advanced Personnel Training Program" (No. 2011-3-068), and the Beijing Municipal Science and Technology Commission (No. Z111107056811041). The authors declare that they have no conflict of interest. The study was approved by the Ethics Committee of the Capital Institute of Pediatrics. Written informed consent was obtained from the parents or guardians of participants in the control group. The Capital Institute of Pediatrics determined informed consent was not needed for the participants in the asthma groups for using the leftover NPA for respiratory virus screening.

\section{Dongmei Chen ${ }^{1}$, Qinwei Song ${ }^{1}$, Runan Zhu ${ }^{1}$, Yuan Qian ${ }^{1}$,} Yu Sun ${ }^{1}$, Jie Deng ${ }^{1}$, Fang Wang ${ }^{1}$, Yaxin Ding ${ }^{1}$, Run Tian ${ }^{1}$, Chuanhe Liu ${ }^{2}$, Wenjing Zhu ${ }^{2}$, Linqing Zhao

1. Laboratory of Virology, Beijing Key Laboratory of Etiology of Viral Diseases in Children, Capital Institute of Pediatrics, Beijing 100020, China

2. Division of Respiratory Diseases, Affiliated Children's Hospital, Capital Institute of Pediatrics, Beijing 100020, China

$\triangle$ Correspondence:

Phone: +86-01-85695576, Fax: +86-01-85610550,

Email: linqingz525@163.com

ORCID:0000-0002-5622-1959
Published online: 6 April 2017

\section{REFERENCES}

Lemanske RF Jr. 2002. Pediatr Allergy Immunol, 13: 38-43.

Jackson DJ, Gangnon RE, Evans MD, et al. 2008. Am J Respir Crit Care Med, 178: 667-672.

Arens MQ, Buller RS, Rankin A, et al. 2010. J Clin Microbiol, 48: 2387-2395.

Balada-Llasat JM, LaRue H, Kelly C, et al. 2011. J Clin Virol, 50: 42-45.

Gharabaghi F, Hawan A, Drews SJ, et al. 2011. Clin Microbiol Infect, 17: 1900-1906.

Selvaraju SB, Selvarangan R. 2012. Diagn Microbiol Infect Dis, 72: 278-281.

Zhao M, Zhu WJ, Qian Y, et al. 2016. Chin Med J (Engl), 129: 15131518.

Zhu RN, Qian Y, Deng J, et al. 2007. Chin J Epidemiol, 28: 1004-1008 (In Chines).

Zhu R, Song Q, Qian Y, et al. 2014. Chin Med J (Engl), 127: 3706-3711.

Asner SA, Rose W, Petrich A, et al. 2015. Clin Microbiol Infect, 21: 264. e1-e6.

McIntyre CL, Knowles NJ, Simmonds P. 2013. J Gen Virol, 94: 1791-1806.

Miller EK, Mackay IM. 2013. J Clin Virol, 57: 291-299.

Moore ML, Stokes KL, Hartert TV. 2013. Curr Opin Immunol, 25: 761-768.

Fawkner-Corbett DW, Khoo SK, Duarte MC, et al. 2016. J Med Virol, 88: 58-63.

Xu R, Shieh YC, Stewart DS. 2017. J Virol Methods, 239: 61-68. 